Artículo de reflexión derivado de investigación
Revista Colombiana de Educación, N. 69 Segundo semestre de 2015 Bogotá, Colombia.

\section{Ciclos de vida como principio activo hacia una escolarización intercultural*} //Life Cycles as an Active Ingredient towards Intercultural Schooling //Ciclos de vida como princípio ativo para uma escolarização intercultural

\section{Recibido: 23/012/2014 Javier Alfredo Fayad Sierra** Evaluado: $04 / 03 / 2015$ 22/04/2015}

Este artículo se basa en el trabajo de investigación del posdoctorado realizado entre el 2010 y el 2012 con el Cinde Universidad de Manizales, Clacso, Universidad Católica de Sao Paulo, Universidad Silva Henriquez.

Profesor de Pedagogía de la Universidad del Valle. Doctor en Educación de la Universidad del Valle, 2006. Licenciado en Educación de la Universidad del Cauca, 1990. Miembro del Grupo Interuniversitario Historia de las Prácticas Pedagógi-

\title{
Resumen
} cas en Colombia. javier.fayad@correounivalle.edu.co

Los ciclos de vida son una referencia sobre el actuar de la naturaleza, y los seres humanos están relacionados directamente con ella, desde sus propios ciclos de vida. Existe una relación arquetípica con esa idea de ciclo de vida, que aparece como un elemento de trascendencia en los pueblos indígenas porque marca parte de los comportamientos que se explican con las cosmovisiones.

Dentro de los saberes ancestrales, que incluyen esta concepción de ciclo de vida, los médicos y las personas especializadas en el conocimiento ancestral expresan sus conocimientos por medio de los "principios activos" de las plantas de saber que manejan las comunidades y de las prácticas de los mismos sabedores. Estas bases de conocimiento se oponen a las del "sistema activo", que es la explicación de las ciencias sobre el actuar de esas plantas de saber, la sistematización del conocimiento que se realizó desde lo antiguo para formular las bases de lo que hoy es la ciencia.

El papel de la escolarización sobre lo que puede ser una repetición o diferencia de los modos escolares explica los modos de imposición de cierto conocimiento y ciertas metodologías sobre el papel de las culturas como diferentes. Nos preguntamos cuál es el papel de la escolarización con respecto a prácticas culturales que no son reconocidas en los modelos de escolarización.

\section{Abstract}

Life cycles are a reference to nature's behavior. Human beings are related directly to it from their own life cycles. There is an archetypal relationship with the concept of life cycle, which appears as a significant element in indigenous peoples because it determines some of the behaviors explained by worldviews. Within ancestral knowledge, including this view of life cycle, medicine men and women as well as experts in ancestral knowledge express their knowledge through the "active ingredients" of knowledge plants familiar to communities, and through those wise men's and women's practices. These knowledge bases are opposed to those of "active system", meaning the explanation science provides for those knowledge plants, the systematization of knowledge carried out in the past to set the ground of today's science.

The role of schooling on what may be a repetition or difference of school ways explains the ways used to impose certain knowledge and methodologies about the role of cultures as different. We asked about the role of schooling concerning cultural practices that are not recognized in schooling models.

\section{Palabras clave}

Ciclos de vida, interculturalidad, pueblos indígenas, ancestral, escolarización

\section{Keywords}

Life cycles, interculturality, indigenous peoples, ancestral, schooling

\section{Palavras chave}

Ciclos de vida, interculturalidade, povos indígenas, ancestral, escolarização 


\section{Resumo}

Os ciclos de vida são uma referência sobre o agir da natureza, e os seres humanos estão relacionados diretamente com ela, desde seus próprios ciclos de vida. Existe uma relação arquetípica com essa ideia de ciclos de vida, que aparece como um elemento de transcendência nos povos indígenas porque marca parte dos comportamentos que se explicam a partir das cosmovisões.

Dentro dos saberes ancestrais, que incluem esta concepção de ciclo de vida, os médicos e as pessoas especializadas no conhecimento ancestral expressam seus conhecimentos por meio dos "princípios ativos", que é a explicação das ciências sobre o agir dessas plantas de saber, a sistematização do conhecimento que se realizou desde o antigo para formular as bases daquilo que hoje é a ciência.

O papel da escolarização sobre aquilo que pode ser uma repetição o diferença dos modos escolares explica os modos da imposição de determinado conhecimento e determinadas metodologias sobre o papel das culturas como diferentes. Por tanto, perguntamos, qual é o papel da escolarização em relação às práticas culturais que não são reconhecidas nos modelos de escolarização.

La forma que se impone como modelo de escolarización es universal, homogeneizadora y promotora de la cultura del consumo. Nos valdremos de ella para hacernos la pregunta sobre lo que pasa con la escolarización en contextos rurales, de otras culturas, otros idiomas, bilingües e interculturales. La inquietud "¿qué pasa...?" se expresa porque al conocer algunas experiencias educativas de pueblos indígenas en Colombia se encuentra que la relación cosmovisión-idioma-pensamiento sugiere la existencia de otras formas de "conocer el conocer", que no son tan escolarizadas en cuanto a sus métodos y contenidos. La pregunta nos lleva necesariamente a pensar una escuela intercultural, desde formas de conocimiento propias del sistema de conocimiento de cada cultura'. En el recorrido por las culturas, en sus expresiones intraculturales al pensar y actuar, se ve que sus conocimientos tienen una sólida base en los ciclos de vida. Este texto indaga cómo los ciclos de vida, desde dentro de las culturas, elaboran, problematizan y dan sentido a los conocimientos propios de cada una de ellas, diferenciándose de los conocimientos generales de Occidente. Haciendo el paralelo con el principio activo de las plantas de conocimiento, se considera que, para llegar a la idea de una escolarización realmente intercultural, es importante tener en cuenta tanto los ciclos de vida como las formas de conocimiento del contexto cultural de cada pueblo indígena.

Lo propio y los conocimientos propios se refieren a formas de asumir procesos que han sido realizados directamente por los pueblos indígenas y que son parte de sus bases de conocimiento y cultura. Es lo propio porque desde la tradición de los mayores, la tradición oral y los aportes de los sabedores (médicos de comunidad), se sabe y afirma que pertenece a la cultura y al mismo tiempo es diferente con respecto a otras culturas. 


\section{Los ciclos de vida}

Las formas de conocimiento milenarias se centran en la explicación que los humanos y las culturas dan de los ciclos de lo que ocurre en su medio. De allí interpretamos en el tiempo y en el espacio esa visión cíclica de las cosas. Los ciclos son parte del proceso que explica cómo actúa la naturaleza: el ciclo del universo, de la Tierra, de la luna, del agua, que nos muestran la referencia del cambio, la circulación, el movimiento. En los seres humanos estos ciclos están presentes en el proceso de conformación de la vida (el nacimiento, el crecimiento y la muerte) y necesariamente se refieren a unos ciclos mayores regulados por la misma relación entre la humanidad y la naturaleza.

La interpretación del contexto, de las cosas que nos rodean como seres humanos hace referencia a esa visión de ciclos en que vivimos. Todo lo que conocemos, lo que existe, tiene unos ciclos de larga duración y de corta duración, gracias a los cuales se establecen formas de equilibrio en la Tierra, en el universo, en los climas, en las plantas, en el cuerpo humano; hasta se habla de biorritmos que definen comportamientos, caracteres y momentos de la vida; se habla de ciclos masculinos y femeninos como diferenciales en los comportamientos y formas de ser.

Históricamente el ser humano, desde lo más antiguo y ancestral, ha tenido la capacidad de observar la naturaleza, comprender el comportamiento de sus ciclos y aplicarlo a su contexto, recreando y creando aspectos culturales de cada tradición y construyendo formas de conocimiento a partir de ellos. De esas formas de ver y pensar los ciclos han surgido las bases de las ciencias naturales, de las matemáticas, de las ciencias básicas, entre otras.

El ciclo de vida es, entonces, una referencia a los ciclos de la naturaleza. Todo lo que existe en el universo se caracteriza con un ciclo rítmico propio y todo lo que inicia surge con un ciclo de desarrollo propio. El sistema solar representa un ciclo mayor y el sistema de la Tierra representa un ciclo menor; esa relación permite definir la relación temporal entre un año y un día. El ser humano tiene un ciclo de existencia dividido en periodos idénticos para todos los seres; se considera normalmente que el ciclo humano comienza con el primer aliento de vida que penetra por la nariz y se cierra con la expiración de ese aliento al concluir la vida (Lewis, 1954, p. 33).

Los teóricos más reconocidos en este aspecto de los ciclos son Carl Gustav Jung, Erik Erikson y Jean Piaget, entre otros, dentro de las denominadas teorías evolutivas o teorías del desarrollo. Jung define una psicología del ciclo vital o psicología de la vida entera, según este autor:

$$
\begin{aligned}
& \text { El niño no es solo un } \\
& \text { ser inicial sino también } \\
& \text { final. El ser inicial era } \\
& \text { antes del hombre, y el }
\end{aligned}
$$


ser final es después del hombre. Psicológicamente, esta afirmación significa que el niño simboliza la naturaleza preconsciente y postconsciente del hombre. Su naturaleza preconsciente es el estado inconsciente de la más tierna infancia, la naturaleza postconsciente es una anticipación per analogium más allá de la muerte. En esta representación, se expresa la naturaleza global de la totalidad humana. (Jung y Kerényi, 2004)

Sassenfeld (2004) considera que Jung es recurrente al mirar las etapas del ciclo de vida desde el papel de la relación entre la conciencia y el inconsciente, relación que está presente durante toda la vida, puede ser corregida y manifestarse en etapas o estadios vitales. Estas etapas se moldean por medio de "imágenes arquetípicas" que afectan el desarrollo de la personalidad y el comportamiento (p. 23).

Consideramos importante esta visión arquetípica de las edades de la vida y del ciclo vital humano jungiano porque nos permite caracterizar, desde el punto de vista de culturas diferentes a la europea, cómo se puede reconocer el sentido arquetípico y simbólico de las etapas de la vida a partir de un referente ancestral que conforma las bases biopsicosociales de los grupos humanos. Jung (en Sassenfeld, 2004) establece la relación entre la evolución filogenética y el desarrollo ontogenético cuando plantea que en cada nueva generación, de alguna manera, se repite la anterior. Es recurrente el hecho de que en el ser humano, el último estadio de desarrollo alcanzado contiene la estructura psíquica entera que sus ancestros desarrollaron en el transcurso de la historia (Sassenfeld, 2004, p. 20).

En los diferentes pueblos indígenas andinos, la cosmovisión plantea una relación con el tiempo que no es lineal, sino circular e integral. No se puede mirar una situación, por ejemplo el ciclo de la agricultura, por fuera del ciclo de los climas, del ciclo de las aguas, del ciclo del territorio, del ciclo de la vida; las relaciones de cada ciclo, su movilidad, no es hacia delante, es hacia arriba, hacia abajo, al lado, adelante, atrás porque es un ciclo que va y vuelve, retorna, recoge, enrolla. El papel de cada persona está ligado a lo que pasa en su entorno desde la visión cosmocéntrica de su cosmovisión. El ser humano no es el centro, como lo plantea la visión de mundo de occidente; al contrario, el ser humano está en relación con las demás cosas, con la naturaleza, con sus ciclos y las situaciones que permiten relacionar 
como fuerza y como energía vital con los otros que componen la totalidad del cosmos.

En estos pueblos existe un tiempo particular que responde a los diferentes ciclos relacionados con el estar y el hacer o estar haciendo de la vida; ese tiempo está ligado con el ciclo de las aguas, con el ciclo de la naturaleza, con el ciclo del territorio donde se relacionan los cambios de los climas, las siembras, los productos y el ciclo de vida particular de cada pueblo que responde a los cambios según el proceso de origen, nacimiento y crecimiento hasta la muerte y la relación que mantienen con el otro mundo, es decir, con los que se van y con los que se quedan.

\section{Ciclos de vida en el pueblo Misak}

En el caso del pueblo Misak, conocido tradicionalmente como Guambiano, Wam-pi, los habitantes de Wampia ${ }^{2}$, su especificación de ciclo de vida es una relación integral, sin jerarquías y sin separaciones entre el derecho-deber de vida misak; los elementos que desde la cosmovisión definen unos instantes del origen misak; el ciclo de vida del misak misak en su entorno de la naturaleza; el ciclo de vida de cada persona como misak

Guambianos es el nombre dado por los visitadores, más en concreto Tomas López, en 1559, para realizar la distribución de las primeras encomiendas (Dagua, Tunubala, Varela, Mosquera, 2005). y las relaciones con el entorno de la estructura comunitaria. Los elementos del ciclo de vida personal en los misak parten de la preconcepción (srusrq) y continúan en la concepción (usri); el nacimiento (srq misak); la niñez (lamq unq); la juventud (kqliunq); la adultez (kqlik); la edad adulta mayor, (kqllimisak); la preparación del viaje espiritual (misak pinap, kuainpabq) y el viaje de regreso (kansrq) (Tombe, Morales \& Tunubala [2008]).

Como ya habíamos planteado, la forma de comprender y vivir este ciclo de vida corresponde a varios elementos que tienen que ver con cómo vive el misak en su contexto completo, con la manera como influye su cosmovisión, en una cosmoexistencia donde están presentes los astros, los páramos, las aguas, las tierras, las plantas, los minerales, los fuegos, los animales, los sistemas agrícolas, los alimentos, las técnicas de vivienda, los vestidos, las músicas, las danzas, los cantos, las pinturas-diseños, los tejidos, las ritualidades, las medicinas, los lenguajes. La totalidad relacionada que produce la forma de vida del misak (Muelas y Tunubala, 2008).

Los dos ciclos anteriores de la persona misak y de sus relaciones con el entorno están igualmente vinculados con las relaciones que desde el territorio, la cosmovisión y la convicción del misak -siguiendo las formas de dignidad, identidad, autonomía y autoridad del origen misak- tienen lugar en lo cotidiano de la vida comunitaria de las 
personas, del núcleo de convivencia, de la familia, de la casa y de la gran casa que es la comunidad en el territorio que permite hablar de un pueblo y una nación (Muelas y Tunubala, 2008).

\section{El principio activo}

Los avances teóricos, prácticos y tecnológicos de la información, la comunicación y el conocimiento demuestran que hoy en día podemos acceder a cualquier tipo de conocimiento sin restricciones y controles ideológicos. En los planos educativo y formativo en el largo plazo, desde las relaciones de conocimiento, se ha planteado como una constante la negación de los pueblos originarios en los medios académicos clásicos de la antropología y la sociología. Esta negación se propone como una diferencia entre pueblos "salvajes" $y$ "civilizados", mientras que en el corto plazo las expresiones políticas y culturales de los pueblos han logrado transformar esa visión hacia una relación de reconocimiento, que en el campo de la formación ha establecido un lugar de diferencia entre el saber ancestral como conocimiento propio, distinto del de la ciencia, que asume el conocimiento como una síntesis cambiante en las denominadas ciencias básicas.

El conocimiento ancestral se basa en la naturaleza, en las relaciones simbólicas y formativas de las "leyes de origen" de los pueblos que explican la relación naturaleza-ser humano, en la perspectiva de comunidad y pueblo, mediada por el conocimiento de los mayores, la historia oral y los médicos (the walas, marӨpik, mamos, tatas, entre otros), quienes tienen la capacidad de "dialogar" con los esenciales: astros, animas, energías entre los tres mundos y sus comunicaciones, que manifiestan situaciones concretas de los ciclos de vida de los pueblos y sus trámites ante la posibilidad de armonizar y equilibrar las relaciones de vida que afectan todo lo que existe en la naturaleza, el medio, los territorios mayores.

Estos diálogos se establecen entre el "principio activo" de cada elemento con el que trabajan los médicos de la comunidad y el "sistema activo", que es la forma como se interpreta el conocimiento aplicado de los sabedores. El "principio activo" está en el conocimiento ancestral de la naturaleza, mientras que el "sistema activo" es la lectura que en el contexto se realiza de las aplicaciones del trabajo de los sabedores de la comunidad. Las plantas, las semillas, las aguas, los páramos, las especies en 
general tienen unas energías que acompañan a cada especie. Desde ese conocimiento, los sabedores producen y reproducen formas de encuentro entre la naturaleza y su aplicación en contexto, por medio del "diálogo" entre "energías" y el "principio activo" que representa el manejo de las esencias.

Mientras tanto, el "sistema activo" lleva a interpretaciones e intervenciones de los contextos del manejo de plantas, especies, semillas, aguas que es interpretado y utilizado por sistemas de información y conocimiento que cuanto más se investigan e intervienen, más se acercan al conocimiento básico de las ciencias, como un tipo de saber sistematizado que interpreta la aplicación de esa relación con la naturaleza.

El conocimiento ancestral centra el trabajo en el "principio activo" de los esenciales de la naturaleza, de las plantas de conocimiento que utilizan los sabedores. En cambio, el conocimiento euroamericano o eurocentrista del modelo de Occidente trabaja con los "sistemas activos" producidos por la química, la botánica y la biología en formas de interpretaciones y conversión de los elementos de la naturaleza, con el interés de hacer que esta se transforme en productos consumibles y comercializables. Lo concreto de esta diferencia es que el conocimiento de Occidente es potente para producir y reproducir los conocimientos básicos, pero no puede trabajar con los esenciales de la naturaleza, que para los sabedores de comunidad se representan en los espíritus de las plantas, de las aguas, de los páramos, minerales que acompañan el "principio activo" de cada esencial de la naturaleza que está vivo en el territorio y es, también, parte del "principio activo" de la naturaleza.

En Colombia hoy se reconoce la existencia de un alto porcentaje de diversidad en ecosistemas y especies. Igualmente se acepta que es productora de diversidades en la palabra, el lenguaje, el idioma, el pensamiento; por la diversidad lingüística, que es diversidad cultural. Reconocer la existencia de un proceso de investigación y formación de esa pluralidad y diversidad en el pensamiento colombiano nos lleva, necesariamente, al mejoramiento y crecimiento académico de los pueblos diversos. Se nos plantea, entonces, en este proceso, la urgencia de que los colombianos abandonemos la formación monolingüe y monocultural que todavía se reproduce en las instituciones educativas, incluso en las universidades. El modelo de sociabilidad que se les ha ofrecido a las culturas diferentes que están presentes en el país nacional es el de integrarse o desaparecer como pueblos, y la escuela sigue siendo una de las herramientas para cumplir tal propósito.

\section{La escolarización intercultural}

La forma escuela crea modelos de repetición del ciclo formativo por edades para significar la relación 
entre la singularidad y la generalidad que cada cultura define como deber, que satisface la necesidad de cada momento del ciclo de vida. Entonces, la repetición y su forma institucional se moldean para cada momento del ciclo de vida; al pasar a otro momento del ciclo se pasa a otra particularidad que produce una singularidad, la cual genera una repetición de una situación diferencial a la repetición que respondía en la necesidad del momento del ciclo de vida anterior. La secuencia y la relación entre ciclo de vida natural, ciclo de vida humano y ciclo de vida escolar se complementan en el modelo de repetición de la forma escuela. La singularidad responde a la producción de una forma humana institucionalizada en la cultura particular, que permite como repetición satisfacer una necesidad humana valorada como propia de la cultura. Esto lleva a afirmar que hay tantas repeticiones institucionales de la formación como diferencias entre los ciclos de vida que plantean las culturas.

La singularidad cultural es posible en la diferencia entre los modelos y las formas de los ciclos de vida de cada cultura. Para cada momento del ciclo de vida de cada cultura aparecerá la repetición de una forma material que sustenta la existencia real material del ciclo de vida. La forma escuela convencional impone una sola forma de repetición de la institución escolar para todas las culturas. Esta manera de hacer las cosas, históricamente, crea negaciones entre las diferencias culturales y produce hegemonías desde una cultura central de orden político institucional sobre cada cultura diferente, generando formas concretas de acomodación de la expresión cultural diferente a la repetición que se coloca más en el orden moral de esa cultura diferencial; desplazando el sentido formativo de la cultura diferencial a un segundo plano, al menos en el orden institucional de la repetición de la forma escuela.

Cuando se repiten las cosas, en este caso el modelo formativo de una cultura, sin tener en cuenta las relaciones singulares de la propia cultura, se produce una ruptura cultural entre el modelo formativo y los ciclos de vida propios de esa cultura particular. Esto significa que se produce un bloqueo conceptual, simbólico y formativo entre lo que se repite como dependencia de un sistema institucional que representa el hacer de una cultura hegemónica y lo que se produce como parte de las representaciones y relaciones propias de la cultura. Esta situación crea diferencias negativas en el proceso valorativo de los resultados de evaluación de la escuela formal aplicados en culturas diferenciales, 
porque se produce un "desconocimiento" de lo propio, que se materializa en un vacío entre lo que se representa como propio y lo que se repite como impuesto. Esta situación de imposición y negación, y de repetición sin sentido dentro del ciclo de vida propio de la cultura, produce según Deleuze (2002) un bloqueo en el proceso de conocimiento de la cultura a la que se le impone un modelo de repetición de una institución, en este caso de formación.

La formación por imposición cultural genera, entre otras, tres situaciones explícitas con respecto a las opciones de reconocimiento de la cultura particular con respecto a la cultura general: una, es la situación de una comprensión finita de aquello que se impone porque no es propio de la cultura y su significación no responde al contexto de los ciclos de vida de la cultura propia. Dos, la nominación del concepto que se impone carece de una naturaleza propia de la cultura y se reproduce en forma aliena$\mathrm{da}$, lo que crea un estado fuera de sí. Tres, las bases de la formación, los conocimientos y los conceptos no están en las bases de la cultura propia y crean una representación con un origen reprimido por su condición de imposición cultural (Deleuze, 2002).

El autor de este estudio considera que en medio de la repetición impuesta aparece la diferencia como una necesidad cultural manifiesta y permanente para poder perdurar y existir como cultura. La existencia de dos culturas, o más, en relación produce situaciones de diferencia permanente, que se concreta en prácticas diferenciales con respecto a aquella repetición que produce la imposición. La existencia y conservación de las referencias de los ciclos de vida (cosmovisiones) al igual que las prácticas propias (prácticas culturales propias) son creadoras simbólicas y conceptuales permanentes que mantienen las diferencias entre comunidades, pueblos o culturas. La situación política de existencia y re-existencia, las celebraciones, los rituales, las tradiciones que se transmiten de generación en generación por medio de las expresiones culturales, el vestido, las músicas, entre otras hacen que la diferencia cultural y el factor de creación sean productores de diferencias, rupturas, desequilibrios, desigualdades; es decir que la misma cultura convencional hegemónica produce rupturas en los ritmos de las personas y de las culturas en cada momento de su ciclo de vida (Deleuze, 2002).

La respuesta ante la repetición que produce diferencia no puede seguir siendo la imposición. Es importante trabajar la relación de diferencia y de reconocimiento de esas diferencias, porque en el tema de formación se pretende que la persona según su ciclo de vida apropie distintas diferencias, dentro de un reconocimiento cultural que parte de la cultura propia 
y que al relacionarse con otras amplíen el campo de relación, de representación y de aprendizaje. Se trata de ampliar y concretar el aprendizaje por diferencia y reconocimiento, antes que por imposición. Este es el problema central que estamos planteando con la forma escuela convencional-oficial, que históricamente construye un modelo de imposición y de repetición hegemónica de un ciclo de vida que pertenece a la cultura eurocentrista-anglosajona y la traslada como hegemonía del conocimiento a las demás culturas, produciendo la negación y la imposición de un solo modelo.

Lo que venimos planteando se centra en la pregunta sobre cómo existe hoy la realidad escolar en los pueblos indígenas, donde el modelo escolar se queda en la repetición y no ha pasado por esa distinción; donde repetir es seguir haciendo lo mismo, donde se somete la repetición a la igualdad y se pone a funcionar el mismo (igual) programa escolar, las mismas asignaturas y actividades. Se requiere desde la perspectiva deleuziana una repetición que remita a la experiencia de lo que vive cada estudiante, y en este caso, también de lo que vive cada cultura. La realidad es que la escuela convencional lleva a que cada estudiante viva lo mismo, donde no se permite vivir como novedad algo que no ha ocurrido. Esta situación hace que el estudiante que pertenece a otras culturas sea excluido y se pretende como desde la cultura "propia" participar de la experiencia de la formación en relación con unos saberes que se encuentran y son revalidados desde los saberes de la cultura.

La escolarización en la visión tradicional, convencional y oficial es la negación histórica del estar, hacer, estar en el mundo que cada pueblo indígena como cultura expresa; aunque para hablar de conocimientos globales y prácticas globales del conocimiento la escolarización ha cumplido un papel de reproductor de esa concepción modernizante de la escuela. Como resultado de la Constitución Política de 1991, el papel pluriétnico y multicultural ubica la escolarización de los pueblos indígenas bajo otras opciones que es preciso que se construyan directamente desde las propias comunidades, donde esta versión del papel de los ciclos de vida pueda influir en la organización de grados y contenidos del modelo de escolarización que se viene aplicando en los pueblos indígenas de Colombia. 


\section{Conclusiones}

Es importante que los modelos de escolarización universales que cumplen con una función de significaciones globales no sean los que "se lleven" a las culturas diferentes del modelo global del conocimiento eurocéntrico de Occidente. Los pueblos indígenas en Colombia y andinos tienen una especial manera de significar su conocimiento desde los ciclos de vida, desde lo más general del cosmos, la naturaleza, el medio ambiente, hasta lo más particular del ciclo de vida de cada miembro de la comunidad. Desde Jung, hay una relación de los ciclos de vida con la remembranza ancestral de las culturas, que está presente para cada nueva generación.

La organización de la cosmovisión, el idioma y el pensamiento establece otras formas de pensar y actuar que son importantes en los modelos de configuración de la escuela y han de ser tenidos en cuenta. La gran dificultad es que la escolarización, como modelo masificado, no reconoce esos otros órdenes de significación, máxime cuando hay conocimientos que no se reconocen ni circulan en formatos escolarizados. Es importante que los conocimientos de los sabedores, de las plantas, de los ciclos diferentes que determinan la vida de los pueblos indígenas sean reconocidos como conocimientos válidos, que se explican desde su función y validez, como un conocimiento eficaz para la pervivencia de estos pueblos en sus contextos.

La escuela produce un tipo de imposición de conocimiento y del modelo formativo que choca con los contenidos y formas de conocimiento de las culturas otras; por eso surgen las preguntas para hablar de educación intercultural: ¿Cómo reconocer diferentes formas de conocimiento? ¿Cómo reconocer el conocimiento ancestral? Dar respuesta a este interrogante es una tarea de toda la sociedad y del Estado, porque la definición de educación intercultural no es "para minorías", forma parte del reconocimiento de la diversidad que funda y amplía el modelo estatal reciente, que se define inclusivo, diverso. Es decir que esta visión educativa de diversas culturas es, primero que todo, una tarea de todos los colombianos y, al mismo tiempo, un factor diferencial de cada cultura con respecto a otras, incluyendo la del colombiano.

El balance que podemos hacer hoy sobre el nivel de implementación de modelos de escolarización bajo los preceptos del saber ancestral, los ciclos de vida y las relaciones interculturales del conocimiento es que las experiencias en las cuales se aplica esta mirada son muy pocas si se las compara con las formas convencionales de actuar de los docentes.

Existen varios modelos organizativos de las comunidades que han emprendido estos cambios y han creado opciones alternativas, como 
las que se describen en el texto Qué pasaría si la escuela..., que recoge más de treinta años de experiencias del CRIC, así como los procesos del pueblo Misak con la construcción del Proyecto Educativo Misak (PEM) para primaria y secundaria.

En estas propuestas hay mucho de creativo en el sentido del papel que cumplen las comunidades como influencia dentro de la escuela, pero como son procesos donde pesa mucho la presencia de la institucionalidad escolar (que todavía controla en gran medida la relación estudiantes-docente), se ven afectadas las prácticas de enseñanza por la falta de materiales didácticos e insumos pedagógicos suficientes para respaldar este tipo de propuestas.

Para el caso de la primaria existen ejemplos donde se cumplen con estas lógicas de escolarización desde la cosmovisión y los ciclos de vida, y el pensamiento desde la condición de origen como pueblos indígenas; pero en secundaria la condición de control desde las políticas institucionales difícilmente deja que estas otras lógicas se implementen.

En algunos casos se considera que la escuela no tiene la función de transmitir la cultura y se plantea que esa es tarea de las familias, especialmente en el aspecto del ciclo de vida, pero cada día se le entrega más responsabilidad formativa a la escuela sobre el quehacer de los niños y niñas.

La gran dificultad es que todavía se tiende a repetir los modelos convencionales de escuela. Esta opción de trabajar más en la diferencia que en la repetición sigue siendo influida por las tradiciones del modelo escolar, de los modos de hacer de los docentes, que todavía no conciben cómo materializar un estar, hacer y saber hacer diferente al modelo convencional.

\section{Referencias bibliográficas}

CRIC. (2004). ¿Qué pasaría si la escuela...? 30 años de construcción de una educación propia. Bogotá: Fuego Azul.

Dagua, A.; Tunubala, G.; Varela, M. y Mosquera, E. (2005). La voz de nuestros mayores. Popayán, Colombia: Cabildo Indígena de Guambia.

Deleuze, G. (2002). Diferencia y repetición. Buenos Aires, Argentina: Amorrortu.

Jung, C., y Kerényi, K. (2004). Introducción a la esencia de la mitología. Madrid, España: Siruela. 
Muelas, J. y Tunubala, F. (2008). Segundo plan de vida y pervivencia misak. Bogotá: OIM.

Sassenfeld, A. (2004). El desarrollo humano en la psicología jungiana. Teoria e implicancias clínicas. Santiago: Universidad de Chile.

Spencer, L. (1954). El dominio del destino con los ciclos de la vida. Crawsfordville, Indiana. USA: Biblioteca Rosacruz.

Tombe, A.; Morales, M. y Tunubala S. (2008). La expresión de afectividad en la familia Misak en el espacio del nachak-fogón. Santander de Quilichao. Universidad Nacional a Distancia (UNAD), Programa de Psicología. 